\title{
GERMAN ASTROPHYSICAL SPACE PROJECTS
}

\author{
W. KLINKMANN \\ Deutsch Agentur für Raumfahrtangelegenheiten \\ Königswinterer Strasse 522-524 \\ 53227 Bonn \\ Germany
}

The fundamental goals of the international astronomical community are the simultaneous use of telescopes for all different wavelength ranges supplemented by cosmic ray and neutrino research.

It is only through the optical and radio window that can celestial objects be observed from the ground; but even those observations are restricted (spreading of the point spread function and wavelength-dependent absorption by the atmosphere; limitation of the VLBI by the earth diameter).

Even in these two special fields, space borne telescopes are in operation (HST, HIPPARCOS) or in development/consideration like Radioastron from RUSSIA, or Quasat by ESA.

Conclusion: undisturbed observation is guaranteed only by space borne telescopes.

This recognition led to many interesting astrophysical space projects created by the international science community and funded by their national and international agencies.

The Sun as a typical main sequence star has been observed by a lot of different space missions, in which Germany was heavily involved, e.g. HELIOS A+B, sounding rocket missions, participation in SAN MARCO, ULYSSES and CGRO (Solar flares observations).

In preparation is the development of experiments for the ESA-mission SOHO.

The exploration of the universe has just begun. The investigation of the Solar System by space probes and the observation of galactic and extragalactic sources by space telescopes have already significantly changed our understanding of cosmic phenomena and of the Universe as a whole.

German space projects are commonly bilateral or international collaborations. After the successful launch and the mission of the X-ray satellite ROSAT, no further national contributions of this size are planned for the future.

What are the German contributions to the in-orbit missions and projects in development starting with highest photon-energies?

1) Gamma Rays: after the participation in the ESA (ESRO) project COS-B, German scientists have also been invited by NASA to deliver one PI-Instrument, COMPTEL (Low Gamma energy range) for the large Compton Gamma Ray Observatory, which has stayed in orbit since April 1991. There is also some participation in the RKA-mission SPECTRUM-X-Gamma with a subsystem for the SODART-Experiment and JET-X.

A new 'Blue Mission' M2 of ESA, INTEGRAL, a Gamma-spectroscopy mission has been selected. Here German scientists plan to participate in the Spectrometer Experiment and the 
X-Ray Monitor.

2) X-Rays: the most important project for DARA in this field is the ROSAT mission which has delivered exciting scientific results since June 1990. Another successful experiment HEXE is mounted on the MIR station. Due to the great success with ROSAT the X-Ray team is also involved in the ESA-XMM-Project with Telescope Scientist (PI status) and by the development of a CCD-detector within the European EPIC collaboration. With the development of X-Ray-Transmission gratings this group is also partner in the NASA-AXAF mission, the third great observatory following HST and CGRO.

3) UV and VISIBLE: by their contribution to the ESA scientific program, Germany is also involved in the Hubble Space Telescope collaboration led by NASA and ESA. Many German scientists have been selected for HST observing time. German scientists are also participating in the data evaluation of the International Ultraviolet Explorer (ESA/NASA/SERC), which is still in orbit in the 15th year of its mission.

The ESA HIPPARCOS astrometry satellite (launch 1989) with important contributions of German scientists, is passing its fourth year of mission successfully. The mission goal has already been achieved.

The launch of an improved ' $1 \mathrm{~m}$ EUV telescope' called ORFEUS for a short term mission with the ASTRO-SPAS free-flying platform to be released and retrieved by the Shuttle is scheduled for the beginning of August 1993.

During D-2 Spacelab mission the super-wide-angle camera GAUSS took 105 pictures of the Milky Way and the surface of the Earth.

For the future, NASA and DARA are discussing a possible German hardware participation in the NASA-FUSE ( 91,2 through $125 \mathrm{~nm}$ ) project. The German contribution could be realized by the delivery of the Wolter telescope system.

In the collaboration with Russia, DARA is trying to find solutions to participate in the SPECTRUM-UV-Mission with a High Resolution Double Echelle Spectrograph (HIRDES). The spectral range should include the wavelengths from 110 to $340 \mathrm{~nm}$.

Another potential cooperation with RKA could be the modification of the GLAZAR II widefield telescope: replacement of the detector camera by a CCD-camera and change of the wavelength range from the UV to the near IR.

4) IR and Radio Range: ESA's Infrared Satellite Observatory (ISO), after some delay and scheduled for launch in 1995, opens up the infrared spectral range for exploration to German scientists who are contributing the ISOPHOTE and a part of the Short Wavelength Spectrometer (SWS) as focal plane instruments.

This year, a new attempt was started for NASA's airborne Stratospheric Observatory for Infrared Astronomy, SOFIA, which will become a new project starting in 1995 or 1996 . A German contribution could be the $2.7 \mathrm{~m}$ telescope system or a major part of it.

Preparations for the ESA Far Infrared Space Telescope cornerstone mission (FIRST) are progressing by developing low noise receivers. A launch is planned for the beginning of the next century.

A smaller participation is also envisaged for the RKA-RADIOASTRON mission.

5) Cosmic Rays: the ASTROMAG project with German participation was originally planned for the US space station Freedom. But in the meantime with the descoping of Freedom no place 
was left for ASTROMAG any longer. Thus the scientists concerned continue their observations by Balloon payloads or from ground (shower experiments).

Simultaneous observations in space with German contributions:

$\begin{array}{lll}\text { CGRO } & - & \text { Gamma rays } \\ \text { ROSAT } & - & \text { X-rays } \\ \text { HST and IUE } & - & \text { UV-visible } \\ \text { ULYSSES } & - & \text { Gamma bursts }\end{array}$

These statistics demonstrate the large opportunities for German astronomers to use the best space borne telescopes in the shorter wavelength ranges. IR and Radio-telescopes will hopefully follow in the near future. 